\title{
Influenza outbreak among students in Ghana: a report from three time points
}

\section{Augustina Angelina Sylverken ( $\nabla$ annan@kccr.de )}

Kumasi Centre for Collaborative Research in Tropical Medicine, Kumasi

\section{Michael Owusu}

Kwame Nkrumah University of Science and Technology

\section{Richmond Yeboah}

Kumasi Centre for Collaborative Research

\section{Philip El-Duah}

Charite Universitatsmedizin Berlin

\section{Richmond Gorman}

Kumasi Centre for Collaborative Research

Joseph Kofi Bonney

University of Ghana Noguchi Memorial Institute for Medical Research

\section{Sherihane Aryeetey}

Kumasi Centre for Collaborative Research

\section{Eric Adu}

Kumasi Centre for Collaborative Research

\section{Yaw Ampem Amoako}

Komfo Anokye Teaching Hospital

\section{Titus Adade}

Komfo Anokye Teaching Hospital

\section{Eric Yeboah}

Komfo Anokye Teaching Hospital

Joseph Bonney

Komfo Anokye Teaching Hospital

\section{Nana Kwame Ayisi-Boateng}

Kwame Nkrumah University of Science and Technology

\section{Christian Drosten}

Charite Universitatsmedizin Berlin

\section{Richard Odame Phillips}

Kwame Nkrumah University of Science and Technology 
Keywords: Influenza virus, outbreak, H1N1, H3N2, Kumasi

Posted Date: September 17th, 2020

DOI: https://doi.org/10.21203/rs.3.rs-34837/v3

License: (c) (1) This work is licensed under a Creative Commons Attribution 4.0 International License. Read Full License 


\section{Abstract}

Background: Influenza viruses are known to be responsible for a number of respiratory disease outbreaks worldwide. While there exists documented information on influenza which allows for effective strategic preparedness activities in industrialized countries, the same cannot be said of developing and underdeveloped countries. It therefore behoves on developing countries to document information on circulating strains of influenza. Such information will enable prioritization of prevention and more targeted preparedness strategies. In this paper, we report on three different outbreaks of Influenza A among Ghanaian students in December 2017, May 2019 and December 2019 in the Ashanti region of Ghana.

Methods: Throat or nasal samples were collected from all students who presented with signs and symptoms of flu with flocked swabs, stored in RNAlater and transported to the laboratories of Kumasi Centre for Collaborative Research (KCCR) at ambient temperature. Following viral RNA extraction, detection of viral nucleic acid of both influenza $A$ and its subtypes including H1N1 and H3N2 was done by real-time reverse transcription Polymerase Chain Reaction (RT-PCR).

Results: A total of 112 samples were taken. An overall Influenza A prevalence of $51.8 \%(95 \% \mathrm{Cl}=42.1$ 61.3) was recorded during the three outbreak time points. Of these, 11 (19.0\%) were confirmed to be $\mathrm{H} 1 \mathrm{~N} 1$ (pdm09) and 47 (81.0\%) were H3N2 strains. All the $11 \mathrm{H} 1 \mathrm{~N} 1$ (pdm09) were identified during the first outbreak. The H3N2 strains were identified during the second and third outbreaks in May 2019 and December 2019 respectively. Our results show that influenza A virus activity was higher during the months of November and December compared to May. Age, contact with infected subjects, temperature, fever within the last 24 hours and headaches showed significant variations ( $p$-value $<0.01$ ) with respect to influenza A. Wald's test revealed that subjects presenting with headaches had about 5 times odds ( $95 \%$ $\mathrm{Cl}=1.4-17.7)$ for influenza $A$ infection compared to subjects without headache $(p=0.014)$.

Conclusion: Our study provides epidemiological information to better understand the incidence and burden of influenza to allow for prioritisation of prevention and control strategies. Such information would aid in controlling and preparing for future influenza epidemics.

\section{Introduction}

Influenza viruses are implicated in highly infectious respiratory disease outbreaks worldwide [1]. The success of influenza viruses in causing seasonal outbreaks year in and year out and eventually pandemics is dependent on the unique ability of the viruses to undergo frequent changes in their surface antigen [2]. As a result, immunity resulting from infection by one influenza virus does not fully protect against antigenic or genetic variants of the same subtype. Annual global influenza epidemics result in an estimated 3 to 5 million cases of severe respiratory illness and up to about 650,000 deaths [3].

The viruses attack all age groups with an estimated attack rate between 5\%-30\% [4]. Although the elderly stand the highest risk of influenza associated mortalities, about $99 \%$ of mortalities resulting from 
influenza are recorded in children, especially those in developing countries [5]. The burden of influenza in developing tropical and sub-tropical countries is likely to be underestimated. A systematic review of 30 years of seasonal influenza epidemiology in these regions revealed that influenza accounts for $10 \%$ of all outpatient visits and about $6.5 \%$ of hospitalizations [6]. However, for most of these developing countries, reports on influenza were considered insufficient to allow for prioritization of prevention, control and preparedness strategies [6]. It is therefore imperative for research laboratories to regularly report data gathered on influenza to aid in planning prevention and control.

In Ghana, reports on influenza viruses have been hospital-based or targeted at at-risk populations. For example, our group has reported on prevalence of $5.7 \%$ and $1.7 \%$ among outpatients [7] and in-patients [8] with acute respiratory infection respectively. Further, our study on respiratory pathogens among Ghanaian pilgrims returning from Mecca had $1.4 \%$ being positive for influenza viruses [9]. Other studies focused on Influenza A H1N1 (pdm2009) and H3N2 reporting rates of $57 \%$ and $26 \%$ respectively [10]. Prior to this report, Influenza viruses had not been implicated in an epidemic or pandemic situation. In December 2017, the Kumasi Academy Senior High School (KUMACA) in the Ashanti region of Ghana was the site of an H1N1 (pdm09) epidemic. This led to the loss of four (4) lives [11]. The Ministry of Health in Ghana previously reported an attack rate of $14 / 1,000$ population with a case fatality rate of $9.1 \%$ [12]. Similarly, in May 2019, there was an outbreak of H3N2 among medical students on a clinical rotation at the Komfo Anokye Teaching Hospital (KATH), the second largest referral hospital in Ghana. Another influenza A outbreak was recorded for KUMACA in December 2019. In this study, we report on samples collected, laboratory diagnosis and results generated during these three independent outbreaks. This report will provide up to date information on currently circulating influenza strains in Ghana and the importance of local laboratory involvement in such situations.

\section{Materials And Methods}

\section{The outbreak setting/study area}

The three outbreaks were centered around three focal areas within the Kumasi Metropolitan Area (KMA). These were KUMACA, Kwame Nkrumah University of Science and Technology (KNUST) and KATH. Outbreak one occurred in December 2017 at KUMACA, outbreak two was in May 2019 at KATH and outbreak three at KUMACA in December 2019.

KUMACA is a senior high school in the Asokore Mampong Municipality of the Ashanti Region. The school has a student population of about 3000 , of which close to $60 \%$ are males. The school has both day and boarding facilities. The boarding students are accommodated in 8 houses namely Prempeh, Sadler, Boakye Dankwa (BoDank), Yaa Achiaa, Akua Nyarko, Jubilee, Nana Boakye Ansah Debrah (NABOAD) and New house. KNUST is a tertiary institution with about 55,000 student size. The KNUST hospital is the university's main health care facility within which is a clinic designated for only students. The hospital, though situated on the university campus, serves the general community surrounding the school. Students from KUMACA are usually referred to the University Hospital, KNUST due to its close proximity 
to the school. KATH is a tertiary healthcare facility which serves as a teaching hospital for the School of Medicine and Dentistry of KNUST. The facility receives referrals from 12 out of the 16 administrative regions of Ghana and has a staff size of about 4000 working in 15 different directorates (13 clinical and 2 non-clinical). The hospital is bordered by communities such as Bantama, Adum, Kejetia and Nhyiaeso. Medical students on clinical rotation are housed in hostels within the hospital environment and in homes in the surrounding neighbourhoods. Figure 1 shows the study areas and the different hospitals where the patients were admitted or visited.

\section{Inclusion criteria}

All students who presented with signs and symptoms of flu were assessed by a Physician and included in the study.

\section{Sample collection}

During the three outbreaks, respiratory samples (throat or nasal swabs) for 112 patients/students were received at the Kumasi Centre for Collaborative Research (KCCR) laboratories at the Kwame Nkrumah University of Science and Technology (KNUST) for testing. The samples were collected with flocked swabs (Copan Group, Brescia, Italy) and stored in 500ml RNAlater (Qiagen, Hilden, Germany) in $1.5 \mathrm{ml}$ tubes (Eppendorf, Regensburg, Germany) and immediately transported to the KCCR laboratories. Some samples were accompanied by case report forms (CRFs). The CRFs examined mainly socio-demographic characteristics, history with animal exposure and treatment outcome of suspected students/patients. All samples taken at the University Hospital, KNUST and the Komfo Anokye Teaching Hospital (KATH) were transported at ambient temperature to the KCCR and tested at its laboratories. External quality control testing of selected samples was performed by Institute of Virology in Charité, Berlin during the first outbreak in December, 2017. For subsequent outbreaks, viral aetiology testing was locally confirmed by KCCR laboratories.

\section{Viral RNA extraction and testing}

Extraction of viral RNA from the samples was done using the Qiagen viral RNA mini kit (Qiagen, Hilden, Germany) according to manufacturer's instructions. Samples were extracted individually with an input volume of $140 \mu \mathrm{l}$ and eluted in $60 \mu \mathrm{l}$ of elution buffer.

\section{Detection of influenza viruses}

All samples were tested for both influenza A and its subtypes including H1N1pdm09 and H3N2. Detection of viral nucleic acid was done by real-time reverse transcription Polymerase Chain Reaction (RT-PCR). Primers and probes used for targeting the viruses and cycling conditions for the PCR run were as previously described for Influenza A and Influenza A subtypes H1 and H3 [10] using the Invitrogen SuperScript III Platinum OneStep buffer system (ThermoFisher Scientific, MA, USA). Testing was performed on a CFX96 Bio-Rad real-time PCR platform with CFX software version 1.6 (Bio-Rad, Singapore). Results of the real time RT-PCR assays were determined by analyzing the cycle threshold (Ct) 
values of the various samples generated by the CFX software. In all tests, samples with $\mathrm{Ct}$ values less than 38 were considered positive and included in the final analysis. All PCR runs were validated by the inclusion of positive and negative controls.

\section{Data management}

Data were double-entered and aggregated into a Microsoft Excel file (version 2019) and cleaned. Statistical analysis was done using R statistical software (version 3.5.1, 2018). Descriptive analysis was performed to determine the frequency and percentages of categorical variables. The association between influenza A detection and other variables was analysed using Fisher's exact test or Chi-square test where appropriate. Variables that were significant were entered into a logistic regression model. Estimates were expressed as Odds Ratio (OR) and 95\% Wald's Confidence interval. A statistically significant result was considered when $p$-value was $\leq 0.05$.

\section{Results}

\section{Socio-demographic and clinical presentations of the study participants}

Samples were received for 133 patients during the three outbreaks. However, case report forms were received for $112(84.2 \%)$ and therefore used in the statistical analysis. The average age of all subjects was $19.2 \pm 3.7$ years. More than half $(62,55.3 \%)$ of the students were below 20 years. The percentage of females was slightly higher than that of males $(51.8 \%$ vs. $48.2 \%)$. No history of animal contact or exposure to swine was reported by the subjects, however, contact with suspected or confirmed infected people was reported $(44,38.6 \%)$. Table 1 gives descriptive information on the study participants and study variables.

Table 1. Basic demographics of study participants and study variables 


\begin{tabular}{|c|c|}
\hline Variable & Description \\
\hline Total number of participants & 112 \\
\hline \multicolumn{2}{|l|}{ Age } \\
\hline Mean (SD) & $19.2(3.7)$ \\
\hline \multicolumn{2}{|l|}{ Gender } \\
\hline Female & $58(51.8)$ \\
\hline Male & $54(48.2)$ \\
\hline \multicolumn{2}{|l|}{ Contact with infected person } \\
\hline No & $70(61.4)$ \\
\hline Yes & $44(38.6)$ \\
\hline \multicolumn{2}{|l|}{ Temperature $\left({ }^{\circ} \mathrm{C}\right)$} \\
\hline Mean (SD) & $37.8(1)$ \\
\hline \multicolumn{2}{|l|}{ Fever during last 24 hours } \\
\hline No & $54(51.9)$ \\
\hline Yes & $50(48.1)$ \\
\hline \multicolumn{2}{|l|}{ Sepsis suspected } \\
\hline No & $91(87.5)$ \\
\hline Yes & $13(12.5)$ \\
\hline \multicolumn{2}{|l|}{ Malaria suspected } \\
\hline No & $87(83.7)$ \\
\hline Yes & $17(16.3)$ \\
\hline \multicolumn{2}{|l|}{ Diarrhoea } \\
\hline No & $98(94.2)$ \\
\hline Yes & $6(5.8)$ \\
\hline \multicolumn{2}{|l|}{ Headaches } \\
\hline No & $31(29.8)$ \\
\hline Yes & $73(70.2)$ \\
\hline
\end{tabular}


For the three outbreaks that occurred, twenty (20) and 40 samples were collected during outbreak 1 and 2 respectively, while 52 samples were collected during outbreak 3 .

The majority (77.6\%) of influenza positive cases were recorded between November to mid-December. Accordingly, outbreaks 1 and 3 which occurred in December had higher proportion of influenza A positive cases than the second outbreak in May. Figure 2 shows the daily distribution of cases during the three outbreak periods.

\section{Distribution of influenza by site}

The majority of the participants were recruited from $\operatorname{KUMACA}(57,50.9 \%)$ followed by $\operatorname{KATH}(40,35.7 \%)$, whilst KNUST recorded the least number of participants $(15,13.4 \%)$. The proportion of influenza positive students in each outbreak varied, with $71.9 \%$ in KUMACA, $46.7 \%$ in KNUST, and $25.0 \%$ in KATH.

\section{Virus detection}

Overall, 58 individuals tested positive for influenza A, giving an overall prevalence of $51.8 \%(95 \% \mathrm{Cl}=$ 42.1-61.3) during the three outbreaks. Of these, 11 (19.0\%) were confirmed to be H1N1 (pdm09) and 47 (81\%) were H3N2 strains. All the $11 \mathrm{H} 1 \mathrm{~N} 1$ (pdm09) were identified during the first outbreak as KUMACA students. The H3N2 strains were identified during the second and third outbreaks in May 2019 and December 2019 respectively.

Of the 58 subjects who tested positive for influenza $A, 10$ individuals who were exposed to infection with influenza A were younger ( 17 years; IQR $=15.2-20.2)$ than those not infected ( 21 years; IQR $=17.8-22.0$ ). Similarly, a higher proportion of positive subjects presented with fever during the last 24 hours of infection $(38 ; 67.9 \%)$ as compared to those without fever $(18 ; 32.1)$. Headache was more common in individuals positive for Influenza A $(51 ; 91.1 \%)$ than those negative for Influenza A $(5 ; 8.9 \%)$.

A comparison of the various variables collected by exposure to influenza A showed significant variations ( $p$-value $<0.01$ ) in age, contact with infected subjects, temperature, fever within the last 24 hours and headaches. Table 2 details the detection of viruses for the different variables considered under the study during the three outbreaks.

Table 2. Association between variables and influenza A detection during the three outbreaks 


\begin{tabular}{|c|c|c|c|c|}
\hline Variable & Negative & Positive & Test stat. & $\mathrm{p}$-value \\
\hline Total & 54 & 58 & & \\
\hline Age & & & Ranksum test & $<0.001$ \\
\hline Median (IQR) & $21(17.8,22)$ & $17(15,20.2)$ & & \\
\hline Gender & & & Chisq. $(1 \mathrm{df})=0.92$ & 0.337 \\
\hline Female & $31(57.4)$ & $27(46.6)$ & & \\
\hline Male & $23(42.6)$ & $31(53.4)$ & & \\
\hline Contact with infected person & & & Chisq. ( $1 \mathrm{df})=22.63$ & $<0.001$ \\
\hline No & $20(37)$ & $48(82.8)$ & & \\
\hline Yes & $34(63)$ & $10(17.2)$ & & \\
\hline Temperature & & & Ranksum test & $<0.001$ \\
\hline Median (IQR) & $37.2(37.1,37.5)$ & $38.2(37.2,38.8)$ & & \\
\hline Fever during last 24 hours & & & Chisq. $(1 \mathrm{df})=17.34$ & $<0.001$ \\
\hline No & $36(75)$ & $18(32.1)$ & & \\
\hline Yes & $12(25)$ & $38(67.9)$ & & \\
\hline Sepsis suspected & & & Chisq. $(1 \mathrm{df})=0.00$ & 1 \\
\hline No & $42(87.5)$ & $49(87.5)$ & & \\
\hline Yes & $6(12.5)$ & $7(12.5)$ & & \\
\hline Malaria suspected & & & Chisq. ( $1 \mathrm{df})=0.51$ & 0.474 \\
\hline No & $42(87.5)$ & $45(80.4)$ & & \\
\hline Yes & $6(12.5)$ & $11(19.6)$ & & \\
\hline
\end{tabular}

Diarrhoea

No

45 (93.8)

53 (94.6) 
Headaches

$<0.001$

No

Yes
$26(54.2)$

$22(45.8)$
$5(8.9)$

$51(91.1)$

\section{Predictors of Influenza Positivity}

In order to determine the factors and symptoms that could predict influenza A positivity, we performed a logistic regression model using variables that were significant at the univariate level of analysis. The variables inserted in the model were having contact with an infected person, age, fever and headaches. All variables were not significant except for having headache. Subjects presenting with headaches have about 5 times odds $(95 \% \mathrm{Cl}=1.4-17.7)$ of being infected with influenza $A$ infection compared to subjects without headache $(p=0.014)$.

\section{Discussion}

The sudden emergence and detection of Influenza A H1N1 (pdm09) in KUMACA in the Ashanti region of Ghana in 2017 was totally unexpected. This tested the preparedness of local health response systems and exposed the weaknesses in these systems as it took close to two weeks and four deaths [11] to confirm aetiology. This revealed the need for strong local capacities for preparedness and response against influenza and emphasised the importance of multisectoral collaboration in epidemic situations to establish aetiology in a timely manner to inform patient management strategies. The importance of a strong local capacity was manifested during the second and third outbreaks in May and December 2019 respectively where aetiology was confirmed within a day.

The different influenza strains detected during the three outbreaks may be attributable to the frequent genetic shift of the surface antigens of the influenza virus and the circulation of these viral subtypes in Ghana. This calls for a concerted effort by all stakeholders to make provisions and enhance preparedness for epidemics by the different strains of the virus at any point in time.

It is apparent from our results that influenza A virus activity may be highest during the months of November and December compared to May. Even though such a firm observation can only be made after surveillance of respiratory viruses all year-round, these months (November and December) are the transition months from the minor rainy season to the dry season in Ghana with moderate intensity of warmth and low humidity. Low temperatures and humidity favour influenza virus infectivity as has been demonstrated by a number of studies [13-16]. The virus keeps maximal infectivity at relatively low humidity while viral inactivation occurs at relatively high humidity and temperature. Consequently, the virus is rendered non-viable after being carried in respiratory droplets for relatively longer periods in high 
humid air. This moderate warmth and low humidity could explain the high influenza activity during the months of November and December. The high humidity of the rainy season around the month of May potentially limits the extent of spread and viability of the virus resulting in the low influenza A activity around that period.

The overall high prevalence of influenza A observed under this study and at the different time points could be due to the relative activity of the influenza virus all year round in tropical regions of Africa as reported by Radin et al after 5 years surveillance of influenza activity in some African countries [17]. Prevalence rates for the first (December 2017) H1N1 (pdm09) and the second (May 2019) H3N2 outbreaks conform to the findings of a three year surveillance report on influenza viruses in Ghana [10] and could represent the prevalence rate of these subtypes in Ghana. The high prevalence rate $(71.2 \%)$ recorded during the December 2019 H3N2 period is characteristic of a temperate pattern of influenza activity between November and January in North African countries and other temperate regions [17-19], and suggestive of the influence of climatic conditions on the transmission of influenza viruses [20]. This high prevalence also points to the endemicity of the virus subtype in Ghana and other West African states [17].

In our analyses, we found that influenza A was more frequently detected in subjects $<20$ years which is consistent with reports that suggest that influenza A, especially H1N1 has a higher attack and relative mortality rates in people under 20 years $[21,22]$. The predominance among this group could be explained by the fact that there is a high level of physical contact and interaction among this group due to overcrowding of students as witnessed in many Ghanaian second cycle schools [23]. Consequently, when one is infected, there is a high rate of spread of the viral infection among the students [20]. This makes them efficient transmitters of the virus and could represent an at-risk group. This study could not establish contact with an infected person as a risk factor $(\mathrm{OR}=0.37)$ of influenza $A$ infection as evidenced by the large proportion of influenza positive cases (70.2\%) who had not had any prior physical contact with an infected person. This is because the virus could be transmitted through infectious droplets and aerosols and as such physical contact with an infected person might not be necessary for viral transmission especially in an epidemic situation [24]. This underlies the effect of overcrowding on the transmission of the virus through infectious droplets and aerosols.

During outbreaks of zoonotic origins, information on contact with animals or wildlife and/or consumption of their meat becomes important $[25,26]$. Our results indicated no history of animal contact. Even though our limited data may not warrant such a conclusion, an observed high prevalence of Influenza A H1N1 (pdm09) in pigs in Kumasi [27] provides hints of the possibility of human-to-swine or vice versa transmission. This underscores the need for systematic swine surveillance in Ghana considering the close contact between humans and livestock especially pigs which are now seen in many developing countries including those of sub-Saharan Africa [28-30].

Although many influenza patients may not present with specific signs and symptoms of illness, the acute onset of respiratory illness is typical for influenza. This includes coughing, fever and headache [31]. 
Although the triad of headache, coughing, and pharyngitis has been found to constitute the major predictors of influenza disease [32], our study found headache as the most important predictor of influenza infection during the outbreaks, as those who presented with headache were five times more susceptible to influenza A infection. This contrasts the findings of Monto et al, (2000) who found fever and coughing to be the most important predictors of influenza disease [33].

Our data comes with some limitations. Key limitation is the lack of data for some of the cases who were recruited. This affects the sample size and statistical power of the study and consequently does not allow for generalisation of findings. Also, information provided by this study does not depict possible seasonal variability as data was only taken during outbreaks and not a year-round surveillance.

In conclusion, our study provides epidemiological information to better understand the incidence and burden of influenza to allow for prioritisation of prevention and control strategies. Information on the current circulating strains would aid in controlling and preparing for the next influenza epidemic and this requires information on the seasonality of influenza, which is sorely lacking. The recent influenza outbreak experience is necessary to develop the next generation of strategic, operational, and tactical plans for any anticipated or unplanned outbreaks.

\section{Abbreviations}

KCCR - Kumasi Centre for Collaborative Research in Tropical Medicine

KNUST - Kwame Nkrumah University of Science and Technology

RT-PCR - Real time Polymerase Chain Reaction

KATH - Komfo Anokye Teaching Hospital

KUMACA - Kumasi Academy Senior High School

KMA - Kumasi Metropolitan Area

CRF - Case Report Form

Ct - Cycle threshold

\section{Declarations}

\section{Ethics approval and consent to participate}

We obtained ethical approval from the Scientific and Ethical review Committee of the School of Medical Sciences, Kwame Nkrumah University of Science and Technology (KNUST) and the Komfo Anokye Teaching Hospital, Kumasi (CHPRE/AP/462/19). Written informed consent was also obtained from each participant at the time of recruitment through signatures and thumbprints. 


\section{Consent for publication}

Not applicable

\section{Availability of data and materials}

The datasets obtained and analysed during the current study available from the corresponding author on reasonable request.

\section{Competing Interest}

Authors declared that they have no competing interests

\section{Funding}

This publication is part of the PANDORA-ID-NET (EDCTP Reg/Grant RIA2016E-1609), funded by the European and Developing Countries Clinical Trials Partnership (EDCTP2) programme which is supported under Horizon 2020, the European Union's Framework Programme for Research and Innovation. The views and opinions of authors expressed herein do not necessarily state or reflect those of EDCTP.

\section{Authors' contributions}

AAS and MO supervised data collection, laboratory analysis and performed the data analysis. RY, P-ED, $\mathrm{RG}, \mathrm{SA}$ and EA conducted the laboratory tests and generated the laboratory data. JKB supported the study with reagents. YAA, TA, ESY, JB and NKAB assisted in sample collection at KATH and KNUST hospitals. AAS drafted the manuscript. $C D$ and ROP supervised the entire work and received funding from EDCTP. All authors have seen, read and approved the final manuscript.

\section{Acknowledgements}

We are indebted to all the study subjects and the study hospitals for allowing this study to be undertaken in their various facilities. We also appreciate the support of all healthcare workers who helped with sampling and recruitment.

\section{Author's information}

Augustina Angelina Sylverken is a Lecturer at the Department of Theoretical and Applied Biology at the Kwame Nkrumah University of Science and Technology (KNUST) and a Research Fellow at the Kumasi Centre for Collaborative Research in Tropical Medicine (KCCR) all in Ghana.

She is interested in the characterization of novel viruses especially those of zoonotic origin.

\section{References}


1. Spreeuwenberg $\mathrm{P}$, Kroneman M, Paget J: Reassessing the global mortality burden of the $\mathbf{1 9 1 8}$ influenza pandemic. American journal of epidemiology 2018, 187(12):2561-2567.

2. Webster RG: Antigenic variation in influenza viruses. In: Origin and evolution of viruses. edn.: Elsevier; 1999: 377-390.

3. Influenza (Seasonal) [https://www.who.int/en/news-room/fact-sheets/detail/influenza-(seasonal)]

4. WHO: Position Paper on Influenza Vaccines. In., vol. 80th YEAR: World Health Organisation 19 AUGUST 2005, 80th YEAR No. 33, 2005, 80, 277-288 http://www.who.int/wer; 2005.

5. Nair H, Brooks WA, Katz M, Roca A, Berkley JA, Madhi SA, Simmerman JM, Gordon A, Sato M, Howie S: Global burden of respiratory infections due to seasonal influenza in young children: a systematic review and meta-analysis. The Lancet 2011, 378(9807):1917-1930.

6. Gessner BD, Shindo N, Briand S: Seasonal influenza epidemiology in sub-Saharan Africa: a systematic review. The Lancet infectious diseases 2011, 11(3):223-235.

7. Annan A, Ebach F, Corman V, Krumkamp R, Adu-Sarkodie Y, Eis-Hübinger A, Kruppa T, Simon A, May J, Evans J: Similar virus spectra and seasonality in paediatric patients with acute respiratory disease, Ghana and Germany. Clinical Microbiology and Infection 2016, 22(4):340-346.

8. Kwofie TB, Anane YA, Nkrumah B, Annan A, Nguah SB, Owusu M: Respiratory viruses in children hospitalized for acute lower respiratory tract infection in Ghana. Virology journal 2012, 9(1):78.

9. Annan A, Owusu M, Marfo KS, Larbi R, Sarpong FN, Adu-Sarkodie Y, Amankwa J, Fiafemetsi S, Drosten C, Owusu-Dabo E: High prevalence of common respiratory viruses and no evidence of Middle East Respiratory Syndrome Coronavirus in Hajj pilgrims returning to Ghana, 2013. Tropical Medicine \& International Health 2015, 20(6):807-812.

10. Bonney JH, Kronmann KC, Lindan CP, Asante IA, Parbie P, Aboagye J, Amankwah J, Odoom JK, Adjabeng M, Nzussouo NT: Virological surveillance of influenza-like illness among children in Ghana, 2008-2010. The Journal of infectious diseases 2012, 206(suppl_1):S108-S113.

11. Ofori-Amoah J, Anokye R, Mensah A, Esinam FA, Yeboah JB, Kontor IK: Influenza A (H1N1) outbreak in the Asokore Mampong Sub-Municipal, Ghana: A case report. Cogent Medicine 2019, 6(1):1648196.

12. Ghana Business News: Health authorities confirm outbreak of H1N1 at Kumasi Academy [https://www.ghanabusinessnews.com/2017/12/08/health-authorities-confirm-outbreak-of-h1n1-atkumasi-academy/]

13. Noti JD, Blachere FM, McMillen CM, Lindsley WG, Kashon ML, Slaughter DR, Beezhold DH: High humidity leads to loss of infectious influenza virus from simulated coughs. PLOS ONE 2013, 8(2).

14. Urashima M, Shindo N, Okabe N: A seasonal model to simulate influenza oscillation in Tokyo. Japanese journal of infectious diseases 2003, 56(2):43-47.

15. Lowen AC, Steel J: Roles of humidity and temperature in shaping influenza seasonality. Journal of virology 2014, 88(14):7692-7695. 
16. Paynter S: Humidity and respiratory virus transmission in tropical and temperate settings. Epidemiology \& Infection 2015, 143(6):1110-1118.

17. Radin JM, Katz MA, Tempia S, Talla Nzussouo N, Davis R, Duque J, Adedeji A, Adjabeng MJ, Ampofo WK, Ayele W: Influenza surveillance in 15 countries in Africa, 2006-2010. The Journal of infectious diseases 2012, 206(suppl_1):S14-S21.

18. Moura FE, Perdigão AC, Siqueira MM: Seasonality of influenza in the tropics: a distinct pattern in northeastern Brazil. The American Journal of Tropical Medicine and Hygiene 2009, 81(1):180-183.

19. Yazdanbakhsh M, Kremsner PG: Influenza in Africa. PLOS Medicine 2009, 6(12):e1000182.

20. He D, Dushoff J, Eftimie R, Earn DJ: Patterns of spread of influenza A in Canada. Proceedings of the Royal Society B: Biological Sciences 2013, 280(1770):20131174.

21. Lemaitre $M$, Carrat $F$ : Comparative age distribution of influenza morbidity and mortality during seasonal influenza epidemics and the 2009 H1N1 pandemic. BMC infectious diseases 2010, 10(1):162.

22. Sullivan KM: Health impact of influenza in the United States. Pharmacoeconomics 1996, 9(3):26-33.

23. Report on free SHS shows overcrowding in schools [https://www.graphic.com.gh/news/education/report-on-free-shs-shows-overcrowding-inschools.html]

24. Tellier R: Aerosol transmission of influenza A virus: a review of new studies. Journal of the Royal Society Interface 2009, 6(suppl_6):S783-S790.

25. Zhou X-N, Bergquist R, Tanner M: Elimination of tropical disease through surveillance and response. In.: BioMed Central; 2013.

26. Tambo E, Ai L, Zhou X, Chen J-H, Hu W, Bergquist R, Guo J-G, Utzinger J, Tanner M, Zhou X-N: Surveillance-response systems: the key to elimination of tropical diseases. Infectious diseases of poverty 2014, 3(1):17.

27. Adeola OA, Olugasa BO, Emikpe BO: Detection of pandemic strain of influenza virus (A/H1N1/pdm09) in pigs, West Africa: implications and considerations for prevention of future influenza pandemics at the source. Infection ecology \& epidemiology 2015, 5(1):30227.

28. Ma W, Kahn RE, Richt JA: The pig as a mixing vessel for influenza viruses: human and veterinary implications. Journal of molecular and genetic medicine: an international journal of biomedical research 2009, 3(1):158.

29. Adeola OA, Adeniji JA: Prevalence of antibodies to influenza viruses among handlers of live pigs at three locations in Ibadan, Nigeria. Vet Ita/ 2010, 46(2):147-153.

30. Adeola O, Olugasa B, Emikpe B: Antigenic detection of human strain of influenza virus A (H3N2) in Swine populations at three locations in Nigeria and Ghana during the dry early months of 2014. Zoonoses and public health 2016, 63(2):106-111.

31. Moore DL, Vaudry W, Scheifele DW, Halperin SA, Déry P, Ford-Jones E, Arishi HM, Law BJ, Lebel M, Le Saux N: Surveillance for influenza admissions among children hospitalized in Canadian 
immunization monitoring program active centers, 2003-2004. Pediatrics 2006, 118(3):e610-e619.

32. Ohmit SE, Monto AS: Symptomatic predictors of influenza virus positivity in children during the influenza season. Clinical infectious diseases 2006, 43(5):564-568.

33. Monto AS, Gravenstein S, Elliott M, Colopy M, Schweinle J: Clinical signs and symptoms predicting influenza infection. Archives of internal medicine 2000, 160(21):3243-3247.

\section{Figures}

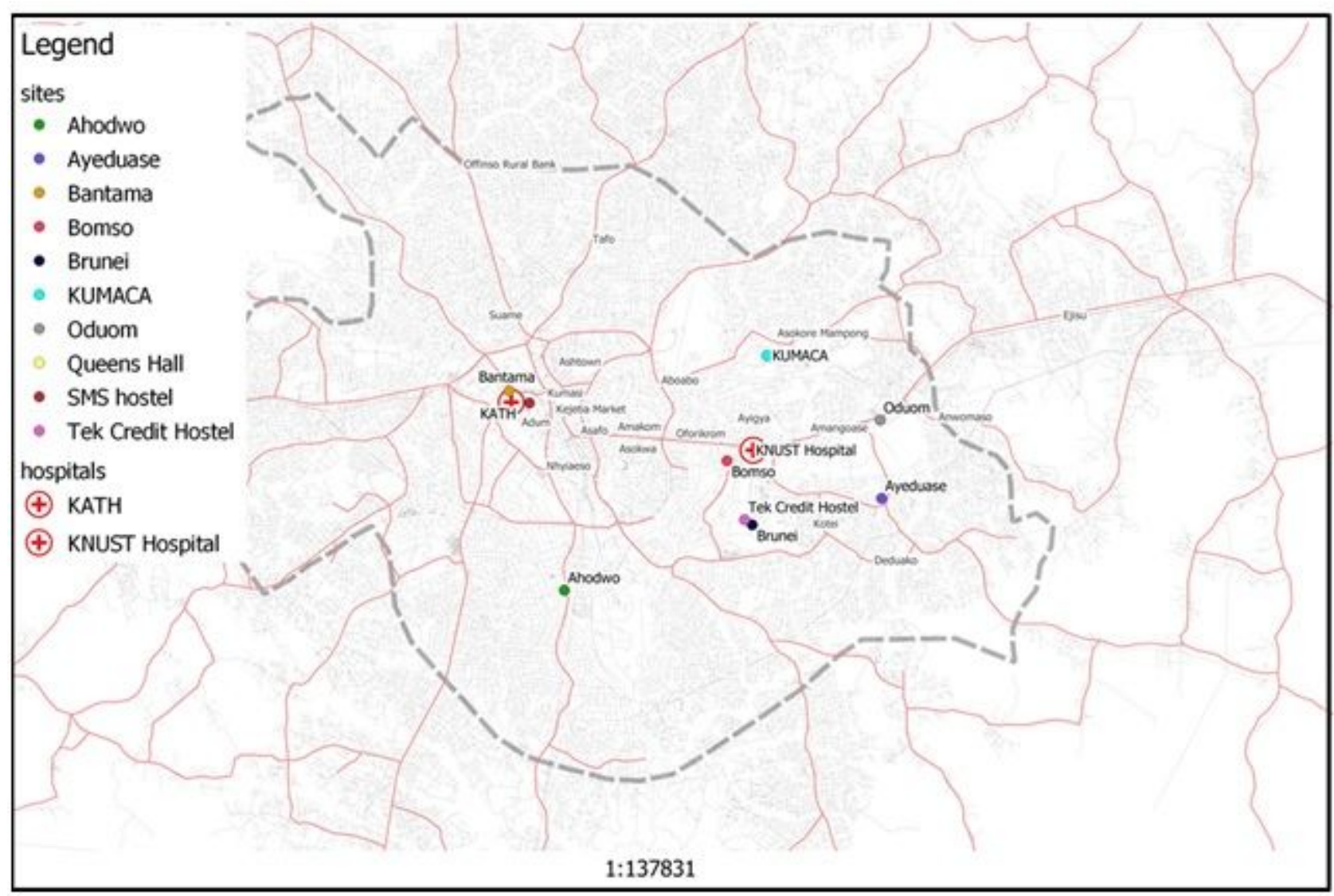

\section{Figure 1}

Map of Kumasi showing the study areas and hospitals of admission of the patients using QGIS desktop version 3.8.3 (QGIS Development Team, Open Source Geospatial Foundation) 

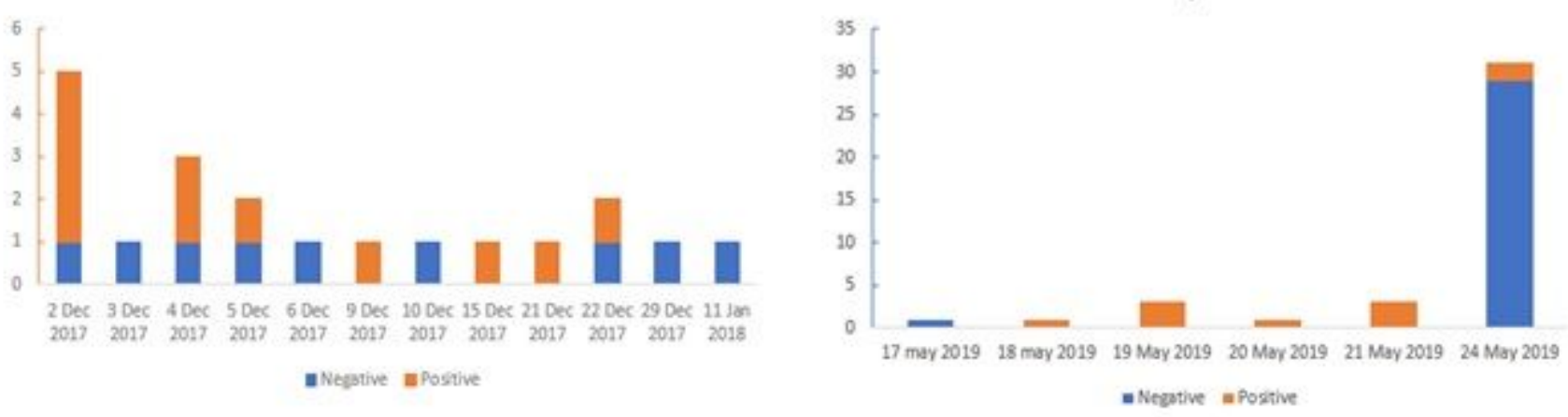

December 2019

$c$

Outbreak pattern

d
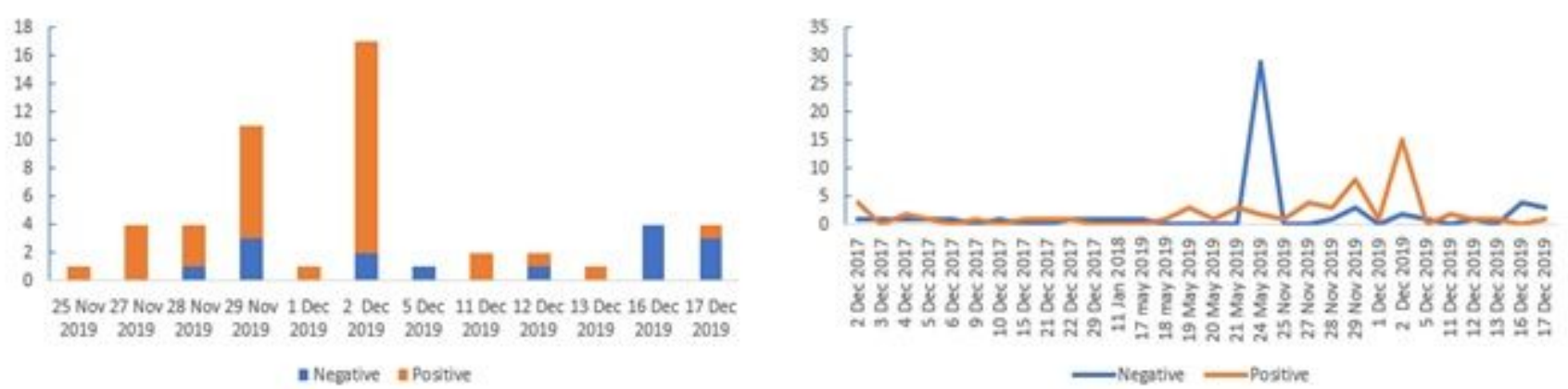

Figure 2

Daily distribution of positive cases among the patients across different days during the outbreak periods. a. Distribution of positive cases during outbreak 1 in December 2017; b. Distribution of positive cases during outbreak 2 in May 2019; c. Distribution of positive cases during outbreak three in December 2019; d. General pattern for the three outbreaks 